\title{
Controle Ótimo para um Sistema Caótico de Lotka-Volterra
}

A. MOLTER e M. RAFIKOV, UNiJUí, Universidade Regional do Noroeste do Estado do Rio Grande do Sul, Mestrado em Modelagem Matemática, Cx.P. 560, 98700-000 Ijuí, RS, Brasil.

\begin{abstract}
Resumo. O propósito desse trabalho é encontrar uma estratégia do controle ótimo que direcione o movimento caótico do sistema de Lotka-Volterra: duas presas e um predador a um ponto fixo desejado. Este problema do controle ótimo é resolvido através da Programação Dinâmica, reduzindo o problema à resolução da equação de Hamilton-Jacobi-Bellman. A solução desta equação é procurada em forma de uma função de Lyapunov logarítmica. Para esse modelo, o algoritmo proposto demonstra a eficiência deste esquema de controle, dirigindo o sistema para o ponto fixo desejado.
\end{abstract}

\section{Introdução}

Nos últimos anos, tem-se observado um grande interesse no controle de sistemas não lineares que exibem comportamento caótico. Os sistemas populacionais, incluindo sistemas presa - predador, também podem apresentar regime caótico [2], [4] . O modelo Lotka-Volterra de duas presas e um predador tem a seguinte forma:

$$
\frac{d x_{i}}{d t}=x_{i}\left(r_{i}-\sum_{j=1}^{3} a_{i j} x_{j}\right), \quad i=1,2,3
$$

onde $x_{1}, x_{2}$ e $x_{3}$ são as densidades da primeira presa, segunda presa e predador, respectivamente. De acordo com Vance [4] e Gilpin [2], esse sistema apresenta comportamento caótico para os seguintes valores dos parâmetros: $r_{1}=r_{2}=-r_{3}=$ $1, a_{11}=a_{12}=a_{22}=a_{23}=0,001, a_{21}=0,0015, a_{13}=0,01, a_{31}=-0,005$, $a_{32}=-0,0005$ e $a_{33}=0$. O que é mostrado na Figura 1 .

O objetivo desse trabalho é eliminar o movimento caótico conduzindo as trajetórias a um ponto fixo desejado, primeiramente através de três funções de controle ótimo, após com aplicação das funções de controle somente nas presas e por fim com aplicação de uma função de controle, somente numa presa. No trabalho anterior [3], para o mesmo modelo, foi considerada somente uma função de controle. 


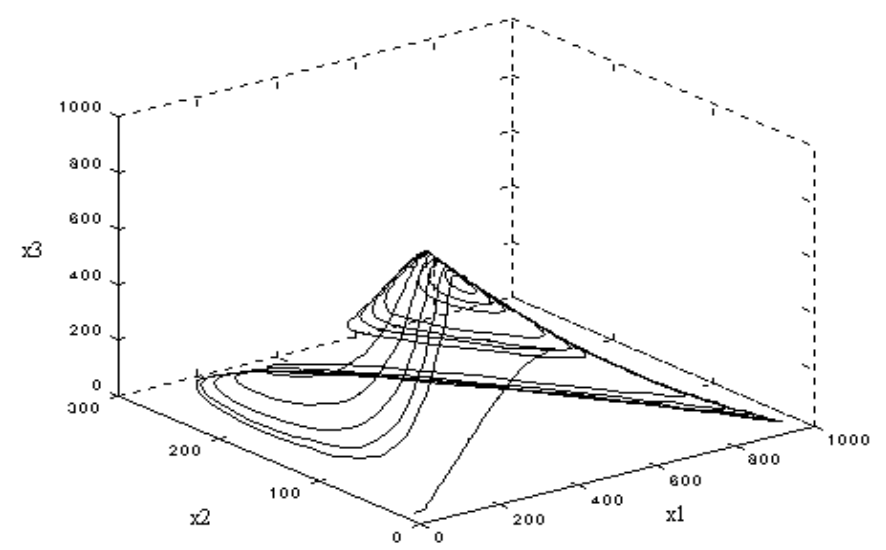

Figura 1: Diagrama de fase do sistema de Lotka - Volterra (1.1).

\section{Controle Ótimo para o Modelo de duas Presas e um Predador do Sistema Lotka-Volterra, apli- cando três Funções de Controle.}

Consideramos o sistema Lotka-Volterra (1.1), incluindo nele três funções de controle $U_{i}(t)$ da seguinte maneira:

$$
\frac{d x_{i}}{d t}=x_{i}\left(r_{i}-\sum_{j=1}^{3} a_{i j} x_{j}+V_{i}+U_{i}\right), \quad i=1,2,3
$$

sendo

$$
V_{i}=-r_{i}+\sum_{j=1}^{3} a_{i j} x^{*}
$$

Para encontrar as funções $U_{i}$, pode ser formulado o seguinte problema do controle ótimo: encontrar as funções de controle $U_{i}$ que transferem o sistema (2.1) do estado inicial

$$
x_{i}(0)=x_{i 0}
$$

ao estado final

$$
x_{i}(\infty)=x_{i}^{*}
$$

minimizando o seguinte funcional:

$$
I[U]=\int_{0}^{\infty}\left[Y^{T} Q Y+l_{1} U_{1}^{2}+l_{2} U_{2}^{2}+l_{3} U_{3}^{2}\right],
$$

onde $Y=\left(\begin{array}{c}x_{1}-x_{1}^{*} \\ x_{2}-x_{2}^{*} \\ x_{3}-x_{3}^{*}\end{array}\right)$ e a matriz $Q=\left(\begin{array}{lll}q_{11} & q_{12} & q_{13} \\ q_{21} & q_{22} & q_{23} \\ q_{31} & q_{32} & q_{33}\end{array}\right)$ é definida positiva. 
De acordo com a programação dinâmica o problema do controle ótimo acima formulado reduz-se à resolução da equação de Hamilton-Jacobi-Bellman:

$$
\min _{U}\left(\frac{d S}{d t}+w\right)=\min _{U}\left(\frac{d S}{d t}+w\right)_{U=U^{0}}=0
$$

onde

$$
w=Y^{T} Q Y+l_{1} U_{1}^{2}+l_{2} U_{2}^{2}+l_{3} U_{3}^{2}
$$

A equação (2.6) é uma equação diferencial parcial não linear. A solução S desta equação deve satisfazer à condição final

$$
S(\infty)=0
$$

Neste trabalho, a função de Bellman $S$ é procurada em forma analítica e, por outro lado, deve satisfazer a equação (2.6) e a condição final (2.8). Ainda tem que fornecer ao sistema (2.1) a estabilidade assintótica. A função de Lyapunov para este sistema, proposta por Volterra [5], é da forma

$$
S\left(x_{1}, x_{2}, x_{3}\right)=\sum_{i=1}^{3} c_{i}\left(x_{i}-x_{i}^{*}-x_{i}^{*} \ln \left(\frac{x_{i}}{x_{i}^{*}}\right)\right)
$$

onde $c_{i}$ são constantes positivas, que podem ser calculadas da equação de HamiltonJacobi-Bellman (2.6), sendo que a função $S$ satisfaz à essa equação e sua derivada $\dot{\mathrm{S}}$, calculada em virtude do sistema (2.1), é

$$
\frac{d S}{d t}=\sum_{i=1}^{3} c_{i}\left(x_{i}-x_{i}^{*}\right)\left(r_{i}-\sum_{j=1}^{3} a_{i j} x_{j}+V_{i}+U_{i}\right), \quad i=1,2,3 .
$$

A parte direita de (2.10) não depende explicitamente de $t$. Isto é conseqüência do fato que a função $S$, dada por (2.9), não depende explicitamente do tempo e sua derivada parcial $\frac{\partial S}{\partial t}=0$.

Levando em conta (2.9) e (2.10), a equação de Hamilton-Jacobi-Bellman (2.6) torna-se

$$
\begin{aligned}
\min _{U}\left\{-c_{1} a_{11}\left(x_{1}\right.\right. & \left.-x_{1}^{*}\right)^{2}-c_{1} a_{12}\left(x_{1}-x_{1}^{*}\right)\left(x_{2}-x_{2}^{*}\right) \\
& -c_{1} a_{13}\left(x_{1}-x_{1}^{*}\right)\left(x_{3}-x_{3}^{*}\right)-c_{2} a_{21}\left(x_{2}-x_{2}^{*}\right)\left(x_{1}-x_{1}^{*}\right) \\
& -c_{2} a_{22}\left(x_{2}-x_{2}^{*}\right)^{2}-c_{2} a_{23}\left(x_{2}-x_{2}^{*}\right)\left(x_{3}-x_{3}^{*}\right) \\
& -c_{3} a_{31}\left(x_{3}-x_{3}^{*}\right)\left(x_{1}-x_{1}^{*}\right)-c_{3} a_{32}\left(x_{3}-x_{3}^{*}\right)\left(x_{2}-x_{2}^{*}\right) \\
& -c_{3} a_{33}\left(x_{3}-x_{3}^{*}\right)^{2}+c_{1} U_{1}\left(x_{1}-x_{1}^{*}\right)+c_{2} U_{2}\left(x_{2}-x_{2}^{*}\right) \\
& \left.+c_{3} U_{3}\left(x_{3}-x_{3}^{*}\right)+w\right\}=0 .
\end{aligned}
$$

A função $U(t)$ não é limitada e pode ser encontrada da condição

$$
\frac{\partial}{\partial U_{i}}\{L(x, U)\}=0
$$


onde através de $\{L(x, U)\}$ é designada a expressão dentre as chaves da equação (2.11).

De (2.12), segue

$$
\begin{aligned}
& U_{1}^{0}=-\frac{c_{1}}{2 l_{1}}\left(x_{1}-x_{1}^{*}\right), \\
& U_{2}^{0}=-\frac{c_{2}}{2 l_{2}}\left(x_{2}-x_{2}^{*}\right), \\
& U_{3}^{0}=-\frac{c_{3}}{2 l_{3}}\left(x_{3}-x_{3}^{*}\right) .
\end{aligned}
$$

Substituindo $U_{i}=U_{i}^{0}$ na equação $(2.11)$, obtém-se

$$
\begin{aligned}
-c_{1} a_{11}\left(x_{1}\right. & \left.-x_{1}^{*}\right)^{2}-c_{1} a_{12}\left(x_{1}-x_{1}^{*}\right)\left(x_{2}-x_{2}^{*}\right) \\
& -c_{1} a_{13}\left(x_{1}-x_{1}^{*}\right)\left(x_{3}-x_{3}^{*}\right)-c_{2} a_{21}\left(x_{2}-x_{2}^{*}\right)\left(x_{1}-x_{1}^{*}\right) \\
& -c_{2} a_{22}\left(x_{2}-x_{2}^{*}\right)^{2}-c_{2} a_{23}\left(x_{2}-x_{2}^{*}\right)\left(x_{3}-x_{3}^{*}\right) \\
& -c_{3} a_{31}\left(x_{3}-x_{3}^{*}\right)\left(x_{1}-x_{1}^{*}\right)-c_{3} a_{32}\left(x_{3}-x_{3}^{*}\right)\left(x_{2}-x_{2}^{*}\right) \\
& -c_{3} a_{33}\left(x_{3}-x_{3}^{*}\right)^{2}-\frac{c_{1}^{2}}{2 l_{1}}\left(x_{1}-x_{1}^{*}\right)^{2}-\frac{c_{2}^{2}}{2 l_{2}}\left(x_{2}-x_{2}^{*}\right)^{2} \\
& -\frac{c_{3}^{2}}{2 l_{3}}\left(x_{3}-x_{3}^{*}\right)^{2}+l_{1}\left(\frac{c_{1}}{2 l_{1}}\left(x_{1}-x_{1}^{*}\right)\right)^{2} \\
& +l_{2}\left(\frac{c_{2}}{2 l_{2}}\left(x_{2}-x_{2}^{*}\right)\right)^{2}+l_{3}\left(\frac{c_{3}}{2 l_{3}}\left(x_{3}-x_{3}^{*}\right)\right)^{2}+Y Q Y=0 .
\end{aligned}
$$

Comparando os termos similares em ambos os lados da equação (2.14), encontrase

$$
\begin{aligned}
a_{12} c_{1}-a_{12} c_{2}+2 q_{12} & =0, \\
a_{21} c_{1}-a_{31} c_{3}+2 q_{13} & =0, \\
a_{23} c_{2}-a_{32} c_{3}+2 q_{23} & =0, \\
a_{11} c_{1}-\frac{c_{1}^{2}}{2 l_{1}}+\frac{c_{1}^{2}}{4 l_{1}}+q_{11} & =0, \\
a_{22} c_{2}-\frac{c_{2}^{2}}{2 l_{2}}+\frac{c_{2}^{2}}{4 l_{2}}+q_{22} & =0, \\
a_{33} c_{3}-\frac{c_{3}^{2}}{2 l_{3}}+\frac{c_{3}^{2}}{4 l_{3}}+q_{33} & =0 .
\end{aligned}
$$

Na simulação numérica, são considerados os seguintes valores: $q_{11}=q_{22}=q_{33}=$ $1, q_{21}=q_{12}=q_{13}=q_{31}=0,0025$ e $q_{23}=q_{32}=0$. Do sistema (2.15), são calculados os valores $c_{1}=0,1786, c_{2}=3,2143, c_{3}=0,6429, l_{1}=0,0080, l_{2}=2,5912 \mathrm{e}$ $l_{3}=0,1033$.

Nas simulações das Figuras 2 e 3 , foi considerado o ponto de equilíbrio $\left(x_{1}^{*}, x_{2}^{*}, x_{3}^{*}\right)$ com valor $(4,10,5)$. 


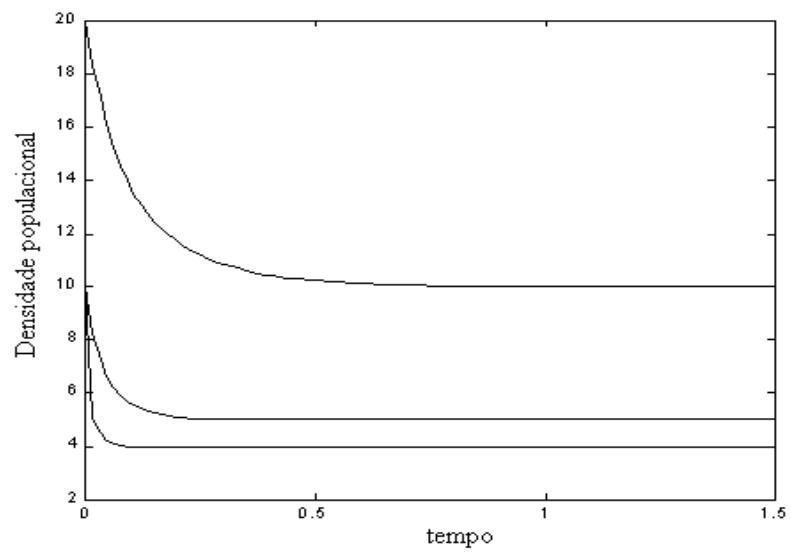

Figura 2: Trajetórias do sistema controlado.

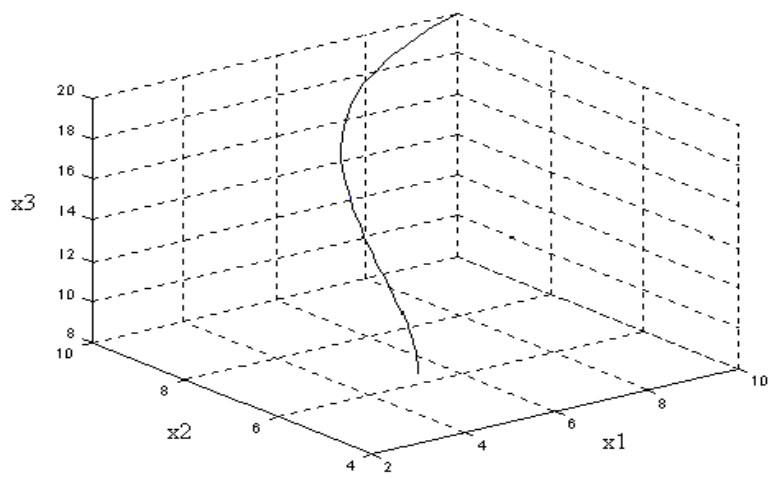

Figura 3: Diagrama de fase do sistema controlado.

\section{Controle Ótimo para o Modelo de duas presas e um predador controlando populações das pre-} sas.

Consideramos o sistema Lotka-Volterra (1.1), incluindo nele duas funções de controle $U_{i}(t)$ da seguinte maneira:

$$
\begin{aligned}
\frac{d x_{i}}{d t} & =x_{i}\left(r_{i}-\sum_{j=1}^{3} a_{i j} x_{j}+V_{i}+U_{i}\right), \quad i=1,2 \\
\frac{d x_{3}}{d t} & =x_{3}\left(r_{3}-\sum_{j=1}^{3} a_{3 j} x_{j}\right)
\end{aligned}
$$

sendo $V_{i}$ de acordo com (2.2).

Para encontrar as funções $U_{i}$ pode ser formulado o seguinte problema do controle 
ótimo: encontrar as funções de controle $U_{i}$ que transferem o sistema (3.1) do estado inicial (2.3) ao estado final (2.4) minimizando o funcional (2.5).

De acordo com a programação dinâmica este problema do controle ótimo reduzse à resolução da equação de Hamilton - Jacobi - Bellman (2.6), onde

$$
w=Y^{T} Q Y+U_{1}^{2}+U_{2}^{2} .
$$

A equação (2.6)é uma equação diferencial parcial não linear. A solução $S$ desta equação deve satisfazer a condição final (2.8).

A função de Bellman $S$ é procurada em forma analítica e, por outro lado, deve satisfazer a equação (2.6) e a condição final (2.8). Ainda tem que fornecer ao sistema (3.1) a estabilidade assintótica. A forma da função de Lyapunov para este sistema presa-predador é (2.9), onde $c_{i}$ são constantes positivas, que podem ser calculadas das condições que a função $S$ satisfaz à equação de Hamilton - Jacobi - Bellman (2.6) e sua derivada $\dot{\mathrm{S}}$, calculada em virtude do sistema (3.1), é:

$$
\begin{aligned}
\frac{d S}{d t}= & c_{i}\left(x_{i}-x_{i}^{*}\right)\left(r_{i}-\sum_{j=1}^{3} a_{i j} x_{j}+V_{i}+U_{i}\right) \\
& +c_{3}\left(x_{3}-x_{3}^{*}\right)\left(r_{3}-\sum_{j=1}^{3} a_{3 j} x_{j}\right), \quad i=1,2 .
\end{aligned}
$$

Levando em conta (3.3) na equação de Hamilton -Jacobi -Bellman (2.6)e derivando parcialmente esta expressão em relação a $U_{i}(2.12)$, obtém-se:

$$
\begin{aligned}
U_{1}^{0} & =-\frac{c_{1}}{2}\left(x_{1}-x_{1}^{*}\right) \\
U_{2}^{0} & =-\frac{c_{2}}{2}\left(x_{2}-x_{2}^{*}\right) .
\end{aligned}
$$

Substituindo $U_{i}=U_{i}^{0}$ na equação de Hamilton -Jacobi -Bellman tem-se:

$$
\begin{aligned}
-c_{1} a_{11}\left(x_{1}\right. & \left.-x_{1}^{*}\right)^{2}-c_{1} a_{12}\left(x_{1}-x_{1}^{*}\right)\left(x_{2}-x_{2}^{*}\right) \\
& -c_{1} a_{13}\left(x_{1}-x_{1}^{*}\right)\left(x_{3}-x_{3}^{*}\right)-c_{2} a_{21}\left(x_{2}-x_{2}^{*}\right)\left(x_{1}-x_{1}^{*}\right) \\
& -c_{2} a_{22}\left(x_{2}-x_{2}^{*}\right)^{2}-c_{2} a_{23}\left(x_{2}-x_{2}^{*}\right)\left(x_{3}-x_{3}^{*}\right) \\
& -c_{3} a_{31}\left(x_{3}-x_{3}^{*}\right)\left(x_{1}-x_{1}^{*}\right)-c_{3} a_{32}\left(x_{3}-x_{3}^{*}\right)\left(x_{2}-x_{2}^{*}\right) \\
& -c_{3} a_{33}\left(x_{3}-x_{3}^{*}\right)^{2}-\frac{c_{1}^{2}}{2}\left(x_{1}-x_{1}^{*}\right)^{2}-\frac{c_{2}^{2}}{2}\left(x_{2}-x_{2}^{*}\right)^{2} \\
& +\left(\frac{c_{1}}{2}\left(x_{1}-x_{1}^{*}\right)\right)^{2}+\left(\frac{c_{2}}{2}\left(x_{2}-x_{2}^{*}\right)\right)^{2}+Y Q Y=0 .
\end{aligned}
$$

Comparando os termos similares em ambos os lados da equação, encontra-se

$$
\begin{aligned}
a_{12} c_{1}-a_{12} c_{2}+q_{12}+q_{21} & =0 \\
a_{21} c_{1}-a_{31} c_{3}+q_{13}+q_{31} & =0, \\
a_{23} c_{2}-a_{32} c_{3}+q_{23}+q_{32} & =0 \\
a_{11} c_{1}-\frac{c_{1}^{2}}{2 l_{1}}+\frac{c_{1}^{2}}{4 l_{1}}+q_{11} & =0, \\
a_{22} c_{2}-\frac{c_{2}^{2}}{2 l_{2}}+\frac{c_{2}^{2}}{4 l_{2}}+q 22 & =0, \\
a_{33} c_{3}+q_{33} & =0 .
\end{aligned}
$$


Nesta simulação numérica são considerados os seguintes valores: $q_{11}=0,251$, $q_{22}=9,001 q_{33}=0, q_{21}=0, q_{12}=0,01, q_{13}=0, q_{31}=0,0051, q_{23}=0,001 \mathrm{e}$ $q_{32}=0$. Do sistema (3.6), são calculados os valores $c_{1}=1$ e $c_{2}=6, c_{3}=1,8$.

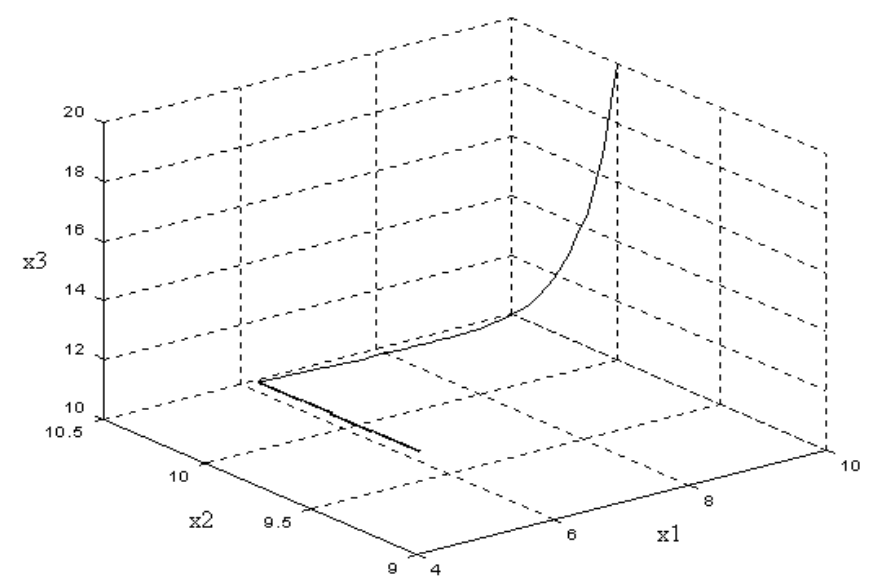

Figura 4: Diagrama de fase do sistema controlado.

Na simulação da Figura 4, foi considerado o ponto de equilíbrio $\left(x_{1}^{*}, x_{2}^{*}, x_{3}^{*}\right)$ com valor $(5,12,7)$.

\section{Controle ótimo para o Modelo de duas presas e um predador controlando a população de uma presa.}

Consideramos o sistema Lotka-Volterra (1.1), incluindo nele uma função de controle $U_{1}(t)$ da seguinte maneira:

$$
\begin{aligned}
\frac{d x_{1}}{d t} & =x_{1}\left(r_{1}-\sum_{j=1}^{3} a_{1 j} x_{j}+V_{1}+U_{1}\right), \\
\frac{d x_{i}}{d t} & =x_{i}\left(r_{i}-\sum_{j=1}^{3} a_{i j} x_{j}\right), \quad i=2,3,
\end{aligned}
$$

sendo $V_{1}$ de acordo com (2.2).

Para encontrar a função $U$ pode ser formulado o seguinte problema do controle ótimo: encontrar a função de controle $U$ que transfere o sistema (4.1) do estado inicial (2.3) ao estado final (2.4) minimizando o funcional (2.5).

De acordo com a programação dinâmica este problema do controle ótimo reduzse à resolução da equação de Hamilton - Jacobi - Bellman (2.6), onde

$$
w=Y^{T} Q Y+U_{1}^{2}
$$


A equação (2.6) é uma equação diferencial parcial não linear. A solução $S$ desta equação deve satisfazer à condição final (2.8).

A função de Bellman $S$ é procurada em forma analítica e deve satisfazer a equação (2.6) e a condição final (2.8). Ainda tem que fornecer ao sistema (4.1) a estabilidade assintótica. A forma da função de Lyapunov para este sistema presapredador é (2.9), onde $c_{i}$ são constantes positivas, que podem ser calculadas da condição que a função $S$ satisfaz a equação de Hamilton - Jacobi - Bellman (2.6), sua derivada $\dot{S}$, calculada em virtude do sistema (4.1), é:

$$
\begin{aligned}
\frac{d S}{d t}= & c_{1}\left(x_{1}-x_{1}^{*}\right)\left(r_{1}-\sum_{j=1}^{3} a_{1 j} x_{j}+V_{1}+U_{1}\right) \\
& +c_{i}\left(x_{i}-x_{i}^{*}\right)\left(r_{i}-\sum_{j=1}^{3} a_{i j} x_{j}\right), \quad i=2,3 .
\end{aligned}
$$

Levando em conta (4.3) na equação de Hamilton -Jacobi -Bellman (2.6) e derivando parcialmente esta expressão em relação a $U_{i}(2.12)$, obtém-se

$$
U_{1}^{0}=-\frac{c_{1}}{2}\left(x_{1}-x_{1}^{*}\right)
$$

Substituindo $U_{1}=U_{1}^{0}$ na equação de Hamilton-Jacobi-Bellman, tem-se

$$
\begin{aligned}
-c_{1} a_{11}\left(x_{1}\right. & \left.-x_{1}^{*}\right)^{2}-c_{1} a_{12}\left(x_{1}-x_{1}^{*}\right)\left(x_{2}-x_{2}^{*}\right) \\
& -c_{1} a_{13}\left(x_{1}-x_{1}^{*}\right)\left(x_{3}-x_{3}^{*}\right)-c_{2} a_{21}\left(x_{2}-x_{2}^{*}\right)\left(x_{1}-x_{1}^{*}\right) \\
& -c_{2} a_{22}\left(x_{2}-x_{2}^{*}\right)^{2}-c_{2} a_{23}\left(x_{2}-x_{2}^{*}\right)\left(x_{3}-x_{3}^{*}\right) \\
& -c_{3} a_{31}\left(x_{3}-x_{3}^{*}\right)\left(x_{1}-x_{1}^{*}\right)-c_{3} a_{32}\left(x_{3}-x_{3}^{*}\right)\left(x_{2}-x_{2}^{*}\right) \\
& -c_{3} a_{33}\left(x_{3}-x_{3}^{*}\right)^{2}-\frac{c_{1}^{2}}{2}\left(x_{1}-x_{1}^{*}\right)^{2} \\
& +\left(\frac{c_{1}}{2}\left(x_{1}-x_{1}^{*}\right)\right)^{2}+Y Q Y=0 .
\end{aligned}
$$

Comparando os termos semelhantes em ambos os lados da equação, encontra-se:

$$
\begin{aligned}
a_{12} c_{1}-a_{12} c_{2}+q_{12}+q_{21} & =0, \\
a_{21} c_{1}-a_{31} c_{3}+q_{13}+q_{31} & =0, \\
a_{23} c_{2}-a_{32} c_{3}+q_{23}+q_{32} & =0, \\
a_{11} c_{1}-\frac{c_{1}^{2}}{2 l_{1}}+\frac{c_{1}^{2}}{4 l_{1}}+q_{11} & =0, \\
a_{22} c_{2}+q_{22} & =0, \\
a_{33} c_{3}+q_{33} & =0 .
\end{aligned}
$$

Para esta simulação numérica são considerados os seguintes valores: $q_{11}=0,251$, $q_{22}=0,001 q_{33}=0, q_{21}=0, q_{12}=0,001, q_{13}=0, q_{31}=0,0025, q_{23}=0,005 \mathrm{e}$ $q_{32}=0$. Do sistema (4.6) são calculados os valores $c_{1}=1$ e $c_{2}=1, c_{3}=1$.

O resultado da simulações do sistema controlado está na Figura 5 . O ponto de equilíbrio $\left(x_{1}^{*}, x_{2}^{*}, x_{3}^{*}\right)$ escolhido para a sistema foi $(5,12,7)$. 


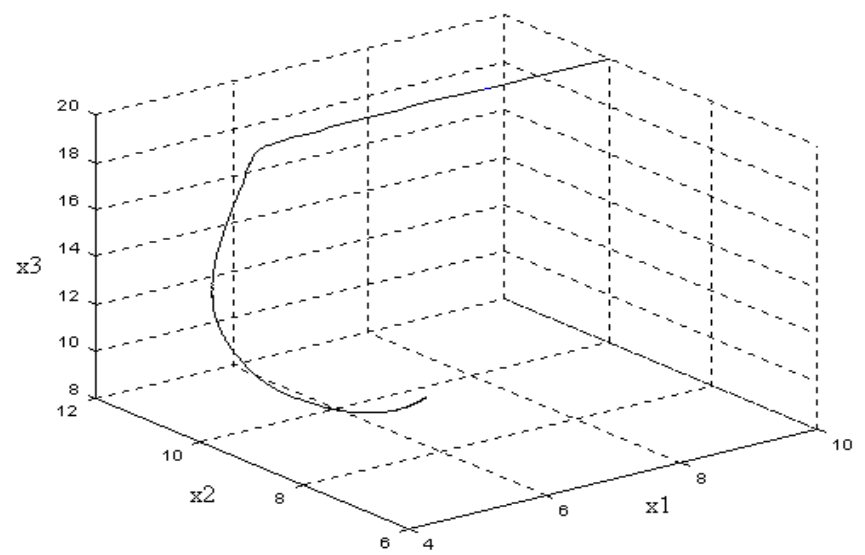

Figura 5: Diagrama de fase do sistema controlado.

\section{Conclusões}

Neste trabalho, consideramos o controle ótimo para o sistema não linear de LotkaVolterra: duas presas e um predador que exibe comportamento caótico. Para resolver o problema de controle formulado foi usada a programação dinâmica. A solução da equação de Hamilton-Jacobi-Bellman foi procurada entre as funções de Lyapunov em forma logarítmica, proposta por Volterra para investigar a estabilidade global dos sistemas deste tipo. Para esse modelo o algoritmo proposto demonstra a efetividade deste esquema de controle dirigindo o sistema para o ponto fixo desejado. Nas três formas propostas, foi atingida a estabilidade assintótica do sistema. Uma das vantagens do algoritmo proposto que ele não requer a linearização das equações, o que é muito importante para sistemas caóticos.

Abstract. The purpose of that work is to find the optimal control strategy that addresses the chaotic movement of the two preys - one predator Lotka-Volterra system to a desired fixed point. This optimal control problem is solved through the Dynamic Programming, reducing the problem to the resolution of the HamiltonJacobi-Bellman equation. The solution of this equation is sought in form of the logarithmic Lyapunov function. For that model, the proposed algorithm demonstrates the effectiveness of this control outline, driving the system for the desired fixed point.

\section{Referências}

[1] R. Bellman, "Dynamic Programing", Princeton, New Jersey, 1957.

[2] M.E. Gilpin, Spiral Chaos in a Predador-Prey Model, American Naturalist, 113 (1979), 306-308. 
[3] M. Rafikov, "Aplicação de Teoria de Controle Ótimo em Dinâmica Populacional ", Anais do I Congresso Temático de Dinâmica, Controle e Aplicações, SBMAC, São Carlos, pp. 311-340, 2002.

[4] R.R. Vence, Predation and Resource Partitioning in one Predator - two Prey, Model Community, American Naturalist, 112 (1978), 797-813; An Evolution, 13 (1998), 325-329.

[5] V. Volterra, "Leçons sur la Théorie Mathematique de la Lutte pour la Vie", Gauthier-Villars, Paris, 1931. 